\title{
Etiology of Fungemia in Neonates: Four Decades of Case Reports
}

\author{
Selma Alves Valente do Amaral-Lopes ${ }^{1}$, José Tavares-Neto ${ }^{2}$ \\ ${ }^{1}$ Department of Pediatrics, Faculty of Medicine of Bahia (FMB), Federal University of Bahia (UFBA), Salvador, Brazil \\ ${ }^{2}$ Department of Medicine, Graduate Program in Heath Science (PPgCS)/FMB/UFBA, Salvador, Brazil \\ Email: selma.lopes@ufba.br
}

Received 28 February 2014; revised 28 March 2014; accepted 4 April 2014

Copyright (C) 2014 by authors and Scientific Research Publishing Inc.

This work is licensed under the Creative Commons Attribution International License (CC BY).

http://creativecommons.org/licenses/by/4.0/

(c) (i) Open Access

\begin{abstract}
Objectives: To compare groups of etiologic agents for neonatal fungemia based on previously published case reports with regard to case outcomes. Methods: Secondary analysis of data from case reports published in scientific literature indexed in Medline, Scopus and LILACS databases, and starting from the year, full texts were registered until the year 2010. Results: Thirty-four species of fungi were isolated in 252 cases reported over a 40-year period. The distribution of fungal groups according to the development level of a case's region of origin was very uneven $(p<0.001)$, with a predominance of $C$. albicans and other fungi (Aspergillus sp.) in more developed countries. However, $73 \%$ of the chi-squared distribution corresponded to differences observed in the frequency of Candida sp. and non-albicans Candida. While the frequency of candidemia by $C$. albicans was progressively declining in the period from 1966 to 2010, reports of non-albicans candidemia and other non-Candida fungi became significantly $(p<0.00001)$ more frequent over the course of the period studied. As for the outcome of death, it was statistically more frequent $(p<0.02)$ in cases where non-albicans Candida or non-Candida fungi were isolated. Conclusion: fungi of the genera Candida and Aspergillus were the etiologic agents most frequently identified in published cases of neonatal fungemia; the outcome of death was more often related to cases where non-albicans Candida or non-Candida fungi were isolated.
\end{abstract}

\section{Keywords}

Neonatal Fungemia, Neonatal Fungal Sepsis, Secondary Data Analysis

\section{Introduction}

Fungal disease in the neonatal period of life almost always presents itself in a serious clinical form, in view of the 
often nosocomial origin, insidious onset and nonspecific clinical profile, and occurrence in an immunologically immature or immunodeficient host, in addition to the lack, quite often, of detection methods available for early etiologic diagnosis [1]-[3]. In relation to these factors, technological advances in recent decades have enabled significant and improving results in the care of newborns, especially those with extremely low birth weight [4]. However, these strategies have also facilitated an increase in fungal infection or colonization, such as hospitalization in intensive care units and the use of various medical procedures (e.g., central venous catheters, invasive mechanical ventilation, parenteral nutrition, the prolonged use and broad spectrum of antimicrobial agents, among others), in addition to the presence of comorbidities [5] [6]. Furthermore, Rodriguez et al. (2010) [7] associated the increased use of fluconazole prophylaxis against infections as another factor determining greater predisposition to fungemia caused by non-albican species of the Candida genus, mainly by selecting for less sensitive species (e.g., C. tropicalis, C. glabrata and C. krusei).

Given the new realities of neonatology, fungemia and invasive fungal disease (IFD) have been deserving of more comprehensive study that is conducted more systematically with improved guidelines, such those recommended by the European Organization for Research and Treatment of Invasive Fungal Infections (EORTC/MSG) [8], which suggested changes to the study of fungal infections in neonates based on the observations of several authors who detected increased isolations of non-albicans Candida species as well as non-yeast cell fungi [9] [10].

In spite of the fact that non-Candida fungi are a cause of fungemia in neonates rarely appears in the literature. This may be due in part to the difficulties of mycological identification. However, in the last decade, there has been an apparent increase in the incidence of cases where non-candida fungi were isolated [10] [11]. Nevertheless, literature lacks any studies which review fungal species isolated in cases of neonatal fungemia. Hence, in this study, we propose to review case reports found in the literature and to compare the etiological groups with outcomes in newborns with fungemia.

\section{Methods}

Case reports of neonatal fungemia published between 1966 and October 2010 were reviewed using secondary data analysis [12] [13]. Cases were found by searching for published cases in indexed sources from the following databases (MEDLINETM, SCOPUS ${ }^{\mathrm{TM}}$, COCHRANE, SciElo, LILACS, Virtual Health Library, CAPES Thesis Database, CAPES Journal Portal via metasearch). We also conducted an active search for cases in the bibliographical references of the selected publications, in specialized texts, among the references of articles related to fungemia, on specialized websites, and in consultations with specialists.

For this purpose, we used terms found in the Medical Subject Heading (MeSH) database from the National Library of Medicine (National Health of Medicine, Bethesda, MD, USA) and in the Descriptors in Health Sciences (DeCS), BIREME (São Paulo, Brazil) to search for cases. We also conducted a structured search using similar search terms based on subject descriptors in a systematized and hierarchical manner by employing specific Boolean operators from the database [14].

Regarding the inclusion criteria, the diagnosis of fungemia, fungal sepsis or IFD was considered a publication fulfilled the defining criteria established by the EORTC/MSG [8]. Only papers written in the Portuguese, Spanish or English languages were included. Cases of publications in which there was no definition of the fungal disease acquired in the neonatal period, written in other languages or using other methodologies (e.g., case series, crosssectional or prevalence studies, cohort studies, case-control studies, clinical trials, editorials, etc.) and studies published prior to the year 1966 or which had not been included in the databases before October 5, 2010 were excluded.

Prior to the pre-selection of articles, a pilot study was conducted to define the MeSH and DeCS keywords, as well as determine the inclusion criteria for case reports of fungemia in the neonatal period, and to determine the best Boolean operators (“and”, "or”, "not”), as described in Amaral-Lopes (2011) [15].

The articles were initially selected by reading the title and summary of the article to determine whether the article concerned case reports. Subsequently, the articles that were pre-selected in this manner, along with those not defining any case reports, were searched for and acquired at websites with access to full texts.

Once the article was reviewed, the inclusion and exclusion criteria were reviewed. After the article was selected, for each case of fungemia described in the article, a data sheet [15] was completed with the following information: demographic, clinical-epidemiological, laboratory, microbiological investigations (i.e., culture, genotyping or other identifying tests, and sensitivity to antifungal agents), treatments, hospital indicators and outcome (discharge 
or death). Initially, the search for and registration of data was performed independently by two researchers and then checked the possible inconsistencies, errors of collection or registration, divergent information or data loss.

Bibliographic references for the selected articles, including those that were eventually excluded, are contained in the paper by Amaral-Lopes (2011) [15].

Data analysis was descriptive, and when a non-parametric test was used (e.g., chi-square or Fisher's exact test), the result was considered statistically significant result when the probability (p) of type I error was less than 5\% (p $<0.05)$.

\section{Results}

A total of 178 articles published in 92 journals between the years 1966 and 2010 were examined, containing 252 cases of neonates diagnosed with fungemia. In the same period, another 59 articles were selected and subsequently excluded: 26 because the cases studied occurred outside the neonatal period; 16 did not include the methodology; 10 were not successfully obtained; and 7 articles had undocumented cases of the fungemia diagnosis.

The 252 cases extracted from 178 articles were distributed over the following years of publication: 10 (4\%) from 1966 to 1979, 61 (24.2\%) from 1980 to 1989, 74 (29.4\%) 1990 to 1999, and 107 (42.4\%) from 2000 to 2010. The majority of cases, corresponding to 59.5\% (150/252), were from the United States of America, Canada and European countries. All eight Brazilian cases (3.2\%) were published after 2000.

The 252 cases were sorted based on their origin in a more developed country or region (North America [except Mexico], Europe [except Turkey], Japan, Australia and New Zealand) or a developing country/region (Asia, Brazil, other countries of the Americas and Africa). Of the cases, 65.1\% $(n=164)$ were from a developed country or region and $34.9 \%(n=88)$ were from a less developed region.

Each of the 178 articles that we found described from 1 to 8 cases, although the majority $(73.9 \%, n=185)$ of the 252 cases were found in articles with one or two cases of newborns diagnosed with fungal sepsis. In 18 (7.1\%) cases, the gender of the recently born baby was not revealed. Of those cases which contained this information ( $\mathrm{n}=$ 234), the majority $(58.1 \%, n=136)$ were male. Of all reported cases $(n=252)$ of newborns with fungemia, in 5 cases (2\%) there was no mention of the outcome (i.e., whether the patient lived or died). The overall death rate was $38.5 \%$ (95/247). There were 230 (91.3\%) valid cases which included both data (gender and clinical outcome); of these, there was a greater frequency of death among boys (59/133, 44.4\%) than among girls (30/97, 30.9\%), a statistically significant difference $(\mathrm{p}<0.04)$.

With respect to mycological investigations after a fungemia diagnosis was suspected, in 7 of the 252 cases (2.8\%), no isolation of any fungal species was documented, while in 16 cases (6.4\%), there was no isolation (result described as negative). Therefore, in 229 cases (90.9\%), there was a record of fungus isolation over the disease course of fungemia, although 2 cases contained no record of the genus or species, and in another 7 cases (3.1\%), two fungi of different genera were isolated. These 227 cases with information about the genera or species are listed in Table 1, which considers the fungal species cited by the author(s) as the primary one. Using this method, a total of 34 species of fungi were found in reports of fungemia in the neonatal period, which were distributed over 19 genera (Table 1). Of these species, the majority (173/227, 76.2\%) corresponded to species of genus Candida, including those $(n=7)$ that were previously classified as Torulopsis glabrata. Of the 173 cases in which the isolated fungus belonged to the genus Candida, the following species were identified: 19 cases (11\%) with no record of a fungal species, 118 (68.2\%) with C. albicans, and 36 (20.8\%) non-albicans. Of the 54 cases (23.8\%) where non-candida species were isolated, 11 cases had fungi of the genus Aspergillus and 9 had fungi of the genus Trichosporon (Table 1).

In the investigation of fungal species, genotyping or another non-morphotintorial identification test was only used in 26 (10.3\%) cases, all of which were found after the year 1995. In 35 (13.9\%) cases, the records showed that the genotype or non-morphotintorial test was not carried out, these articles being published between the years 1968 and 2010. However, in the majority of reports $(n=191,75.8 \%)$ there was no record of this type of investigation.

In the majority of cases $(n=192,76.2 \%)$ there was no record of whether research on the sensitivity of the isolated fungi to antibiotics or antifungal chemotherapy was performed. In only six cases (2.4\%) was there a record showing that the test had not been performed. Sensitivity testing was performed in 54 (21.4\%) cases, 45 (83.3\%) of which were sensitive to amphotericin B, and in 9 cases (16.7\%) the patients were resistant. The following resistant species were isolated: C. albicans $(\mathrm{n}=2)$, C. parapsilosis $(\mathrm{n}=2)$, C. haemulonii $(\mathrm{n}=1)$, C. krusei $(\mathrm{n}=1)$, C. lusitaniae $(\mathrm{n}=1)$, C. tropicalis $(\mathrm{n}=1)$ and T. asahii $(\mathrm{n}=1)$. 
Table 1. Fulgal species responsible for fungemia in neonatal cases reported in the literature (1966-2010).

\begin{tabular}{|c|c|c|}
\hline Espécie & $\mathbf{N}$ & $\%$ \\
\hline Absidia corymbifera & 1 & 0.5 \\
\hline Acremonium strictum & 1 & 0.5 \\
\hline Aspergillus flavus & 1 & 0.5 \\
\hline Aspergillus fumigates & 7 & 3.2 \\
\hline Aspergillus niger & 1 & 0.5 \\
\hline Aspergillus sp. & 2 & 0.9 \\
\hline Aureobasidium pullulans & 1 & 0.5 \\
\hline Bipolaris sp. & 1 & 0.5 \\
\hline Bipolaris spicifera & 1 & 0.5 \\
\hline Candida albicans & 111 & 50 \\
\hline Candida dubliniensis & 1 & 0.5 \\
\hline Candida glabrata & 3 & 1.4 \\
\hline Candida guilhermondii & 2 & 0.9 \\
\hline Candida haemulonii & 4 & 1.8 \\
\hline Candida krusei & 1 & 0.5 \\
\hline Candida lipolytica & 1 & 0.5 \\
\hline Candida lusitaniae & 3 & 1.4 \\
\hline Candida parapsilosis $^{*}$ & 18 & 8.1 \\
\hline Candida pilliculosa & 1 & 0.5 \\
\hline Candida pseudotropicalis & 1 & 0.5 \\
\hline Candida sp. & 19 & 8.6 \\
\hline Candida tropicalis & 1 & 0.5 \\
\hline Cryptococcus laurentii ${ }^{\dagger}$ & 3 & 1.4 \\
\hline Curvularia lunata & 1 & 0.5 \\
\hline Hansenula anomala & 2 & 0.9 \\
\hline Histoplasma capsulatum & 1 & 0.5 \\
\hline Kodamaea ohmeri & 1 & 0.5 \\
\hline Malassezia furfur & 6 & 2.7 \\
\hline Paecilomyces lilacinus & 1 & 0.5 \\
\hline Phialemonium obovatum & 1 & 0.5 \\
\hline
\end{tabular}




\begin{tabular}{|c|c|c|}
\hline Pichia anomala ${ }^{\ddagger}$ & 3 & 1.4 \\
\hline Pichia fabiani & 2 & 0.9 \\
\hline Rhizopus arrhizus & 1 & 0.5 \\
\hline Rhizopus indicus & 1 & 0.5 \\
\hline Rhizopus sp. & 3 & 1.4 \\
\hline Saccharomyces cerevisiae & 3 & 1.4 \\
\hline Torulopsis glabrata" & 7 & 3.2 \\
\hline Trichosporon asahii & 3 & 1.4 \\
\hline Trichosporon beigelii ${ }^{5 \pi}$ & 5 & 2.3 \\
\hline Trichosporon sp. & 1 & 0.5 \\
\hline Total & 227 & 100 \\
\hline
\end{tabular}

${ }^{(*)}$ one case with Rhizopus sp.; ${ }^{(\dagger)}$ one case related with $R$. indicus and other with C. albicans; ${ }^{\ddagger)}$ one case with $C$. parapsilosis; ${ }^{(\S)}$ two cases with $C$. albicans; ${ }^{(1)}$ the same C. glabrata; and ${ }^{(\text {()) }}$ one case with C. albicans.

Excluding the five cases which offered no description of the clinical outcome and the cases which did not identify the genus or species of the fungus $(n=30)$, a similar distribution was noted $(p>0.55)$ between those patients who survived (17/152, 11.2\%) and those who died (13/95, 13.7\%). There was an uneven distribution ( $<<$ 0.009) of these cases over the following four approximately decade-long periods: 1966-1979 (5/10), 1980-1989 (5/61), 1990-1999 (14/74) and 2000-2010 (6/107), with frequencies of 50\%, 8.2\%, 18.9\% and 5.6\%, respectively.

The seven cases of mixed fungal infection (Table 2) were evenly distributed (Fisher's exact test, $\mathrm{p}>0.53$ ) between the cases in which the patient lived (4/135, 3\%) and died (3/82, 3.7\%). As shown in Table 2, only the valid cases for each analysis were considered, and the fungi were grouped into the following 4 categories: C. albicans, Candida sp., non-albicans Candida fungi and other genera. As shown in Table 2, within these 4 categories, the gender distribution of newborns was similar $(p>0.53)$, whereas the distribution based on the degree of development of a case's region of origin was very uneven $(\mathrm{p}<0.001)$. The predominance of isolates of $C$. albicans and fungi from other genera were identified in developed countries or regions (Table 2). However $73 \%$ of this total (according to chi-square analysis) corresponded with the frequencies of Candida sp. and non-albicans Candida. Nevertheless, the frequency of candidemia by C. albicans was progressively declining in the period from 1966 to 2010, but reports of non-albicans candidemias and other non-Candida fungi were significantly more frequent $(\mathrm{p}<0.00001)$. The outcome of death was statistically more frequent $(\mathrm{p}<0.02)$ in cases where Candida sp. was isolated and in cases involving other non-Candida fungi.

\section{Discussion}

Neonatal IFD, more commonly known as neonatal fungemia, is as yet poorly understood. In this study, IFD during the neonatal period was investigated using a methodology which made it possible to group the characteristics of the entity and related agents such as non-candida fungi, which would have been difficult to study using any other methodology. However, one of the methodological limitations of this study that should be considered when interpreting our results is the paucity of data recorded by the study authors. Nonetheless, understanding the characteristics of the cases described in the literature and the gaps in these reports could serve as the basis for future studies or clinical trials.

In this study, we included 178 articles that were selected from a historical period of 40 years. The information contained therein enables one to characterize how these publications have evolved over four decades, there having been observed an increasing number of publications of reporting cases of neonatal fungemia, especially as of the 1990 's and more frequently (65.1\% vs. $34.9 \%)$ in the more developed countries or regions of the world. This 
Table 2. Fungal groups divided according to gender, case region, publication year and outcome.

\begin{tabular}{|c|c|c|c|c|c|c|}
\hline & & \multicolumn{4}{|c|}{ Fungal Group $-\mathrm{n}(\%)$} & \multirow{2}{*}{ TOTAL } \\
\hline \multicolumn{2}{|c|}{ Variable } & C albicans & Candida sp & Non-albicans & Other fungi & \\
\hline \multirow{3}{*}{ Gender } & Female & 37 (42.) & $8(9.2)$ & $18(20.7)$ & $24(27.6)$ & $87(100)$ \\
\hline & Male & $62(52.6)$ & $9(7.6)$ & $18(15.2)$ & $29(24.6)$ & $118(100)$ \\
\hline & Statistic & \multicolumn{3}{|c|}{$\chi^{2}=2.21 ;$ g.l $=3 ; P>0.53$} & & \\
\hline \multirow{3}{*}{ Economic Region } & Undeveloped & $31(38.3)$ & $12(14.8)$ & $23(28.4)$ & 15 (18.5) & $81(100)$ \\
\hline & Developed & 76 (53.9) & $7(5)$ & $18(12.8)$ & $40(28.3)$ & $141(100)$ \\
\hline & Statistic & \multicolumn{3}{|c|}{$\chi^{2}=17.26 ;$ g.l $=3 ; P<0.001$} & & \\
\hline \multirow{5}{*}{ Publication Year } & $1966 \longmapsto 1979$ & $4(80)$ & $1(20)$ & 0 & 0 & $5(100)$ \\
\hline & $1980 \longmapsto 1989$ & 39 (69.6) & $6(10.7)$ & $4(7.1)$ & $7(12.5)$ & $56(100)$ \\
\hline & $1990 \longmapsto 1999$ & $31(51.7)$ & $6(10)$ & $11(18.3)$ & $12(20)$ & $60(100)$ \\
\hline & $2000 \longmapsto 2010$ & $33(32.7)$ & $6(5.9)$ & $26(25.7)$ & $36(35.6)$ & $101(100)$ \\
\hline & Statistic & \multicolumn{4}{|c|}{$\chi^{2}$ linear distribution $=18.54$; g.l $=1 ; P<0.00001$} & \\
\hline \multirow{3}{*}{ Outcome } & Death & $34(41.5)$ & $11(13.4)$ & $11(13.4)$ & $26(31.7)$ & $82(100)$ \\
\hline & Live & $72(53.3)$ & $7(5.2)$ & $30(22.2)$ & $26(19.3)$ & $135(100)$ \\
\hline & Statistic & \multicolumn{4}{|c|}{$\chi^{2}=1.03 ;$ g.l $=3 ; P<0.02$} & \\
\hline
\end{tabular}

finding is similar to that described by Figueiredo et al. (2006 and 2007) [16] [17], and may be the result of more periodicals being published in the more developed countries, greater facility with the publication language, greater scientific and technological development, among other determinants.

As for the etiology of fungal disease in the cases selected, different genera and species of fungi were reported, which were responsible for the processes of colonization and infection of newborns described in this study [15]. The 34 species of fungi isolated in the case studies were distributed between 19 genera and nine taxonomic orders [18].

No similar study was found in the literature, and it is noteworthy that the number of isolates would likely have been much higher if all reports had sought to identify the fungal colonization and documented the result [15]. Furthermore, although the majority of cultures recorded obtained a positive result (fungal isolation), other possible sources of infection were poorly investigated [15]. Apart from the difficulties inherent in mycological diagnosis, for most tertiary health care units, the absence of a specific protocol to investigate IFD in the neonatal period is another factor that may explain these gaps in the literature. However, this study permitted, perhaps for the first time, a more complete description of fungal etiologies in cases of neonatal fungemia.

The elevated prevalence of the genus Candida (76.2\%) observed in cases of fungemia was similar to that noted in other studies [5] [11]; note, however, the prevalence of non-Candida fungi, 23.8\%, that was observed in this study, including those of the genus Aspergillus, among others. Although the occurrence of neonatal IFD by fungi of the genus Aspergillus is rarely described in the literature [9], this apparent error may be due to the fact that there are more records published in the form of case reports; thus, such cases may not have been published in academic texts or reviews. Therefore, this kind of gap could be filled by means of the methodology applied in this study. It has revealed an increase in the frequency of cases of non-Candida fungi and non-albicans Candida, as well as an increase in cases during the last decade, possibly as a consequence of improvements in diagnostic support and of increased exposure of the hosts.

Also, the results of this study are consistent with a study conducted by Gniadek et al. (2010) [19] with respect to non-Candida species as an etiology for IFD. The authors found an increased prevalence of environmental contamination and colonization of individuals working with infants in pediatric and neonatal intensive care units, primarily by cytotoxic strains of Aspergillus spp., in particular A. fumigatus. The study found $20 \%$ colonization on 
the hands of health care professionals. In future, the development of new, more sensitive diagnostic methods may permit the identification of these more uncommon fungal agents during the neonatal period [3] [9] along with the taxonomic identification of these and other fungal species. In the cases examined here, only $10.3 \%$ underwent genotyping or other analyses in order to better characterize the fungal species and also consider the vast complexity of the taxonomy of fungi [18]. Clark et al. (2004) [1] showed that although the rates of sepsis acquired in a nosocomial environment increase when the degree of prematurity is higher and the birth weight lower, there is not a more sensitive diagnostic procedure that can improve the ability of health professionals to determine whether there truly is an infection in the bloodstream. Therefore the situation as it currently stands may be associated with a greater number of unnecessary treatments, delay in the diagnosis of sepsis, and increased mortality rate.

The majority of IFD cases were considered confirmed by the identification of a pathogen in naturally sterile areas; yet very few studies have evaluated the sensitivity of fungal isolates to antifungal antibiotics. It is, however, necessary to change this practice, which is incompatible with the increasingly frequent use of antifungal prophylaxis [20] [21], and the emergence of strains resistant to the most common antifungal drugs, but also because of the increased incidence of fungal diseases and the increasing number of infections with non-Candida species.

In this study, of the few cases studied $(n=54), 16.7 \%$ of isolates were resistant to amphotericin B. Recently, Yildiran et al. (2010) described [22] different standards of response to therapies being correlated with the newborn's birth weight. For this reason, the authors emphasized the growing need for research concerning the sensitivity of the fungus to antifungal medications, with the objective of not only enhancing treatment planning but also aiding in the correct and early detection of the most fluconazole-resistant strains, since this is the primary medication used in IFD prophylaxis as well as an adjunctive medication with other drugs [23]. This notwithstanding, the cases studied here, amphotericin B was the most commonly used antifungal in over the past four decades [15], despite the emergence of various new antifungal medications [24], which is similar to the current proposal for IFD treatment [23] in the neonatal period.

The IFD-related fatality rate (38.5\%) observed in this study was much higher than those found in the studies by Saiman et al. (2000) [25] and Rodriguez et al. (2006) [26], which cited rates of $22 \%$ and $20 \%$ respectively. Their findings also diverge from other studies which demonstrated rates ranging from $41 \%$ to $76 \%$ [2] [5]-[7]; however, in these most recent studies, the rates were related to fungemia but not attributed to it.

The highest mortality rate was related to cases of non-Candida fungi and non-albicans Candida, which could be related to the pathogenic mechanisms of these fungi [7] [27], as well as the delay in their identification [28], resulting in a delay initiating treatment and increased lethality [29].

Even after considering the elevated global mortality rate observed among premature babies [4], one must consider the hypothesis that the newborns, including those in this study who died at the earliest age, also had more serious diseases during the neonatal period. Thus, fungal sepsis would be an additional aggravating factor to these other morbidities. However, the higher mortality rate among male neonates ( $44.4 \%$ vs. $30.9 \%)$ in the cases examined here, independent of comorbidity or early death, is similar to reports in the literature, which have noted the greater number of preterm births of boys and the apparent biological disadvantage associated with the male gender, which requires further research [1]-[4] [30].

\section{Conclusion}

Therefore, it can be concluded that the leveduriform fungi of the Candida genus, primarily C. albicans and fungi of the genus Aspergillus, are the etiologic agents most frequently identified in published neonatal fungemia cases. In addition, the rate of mortality was found to be as high as those recorded in the literature, in which the outcome of deaths was more prevalent in cases of non-albicans Candida and non-Candida fungi.

\section{Financial Support}

CAPES-Masters fund.

\section{Acknowledgements}

At Thalma Clívia Moraes Ferreira de Melo, Resident (R3) of the Residency Program in Neonatology, Maternity Reference Professor José Maria de Magalhães Netto, the manual collection of data and valuable suggestions.

To the Academic courses of Medicine, FMB/UFBA: Simone Rocha Araújo, Duilho Pablo Leon de Oliveira, 
Ícaro Santos Oliveira and Sérgio Figueiredo Camera for electronic data collection and checking of selected article.

\section{References}

[1] Clark, R., Powers, R., Withe, R., Bloom, B., Sanchez, P. and Benjamin Jr., D.K. (2004) Nosocomial Infection in the NICU: A Medical Complication or Unavoidable Problem? Journal of Perinatology, 24, 382-388. http://dx.doi.org/10.1038/sj.jp.7211120

[2] Benjamin Jr., D.K., Stoll, B.J., Fanaroff, A.A., McDonald, S.A., Oh, W., Higgins, R.D., et al. (2006) Neonatal Candidiasis among Extremely Low Birth Weight Infants: Risk Factors, Mortality Rates, and Neurodevelopmental Outcomes at 18 to 22 Months. Pediatrics, 117, 84-92. http://dx.doi.org/10.1542/peds.2004-2292

[3] Blyth, C.C., Chen, S.C., Slavin, M.A., Serena, C., Nguyen, Q., Marriott, D., et al. (2009) Not Just Little Adults: Candidemia Epidemiology, Molecular Characterization, and Antifungal Susceptibility in Neonatal and Pediatric Patients. Pediatrics, 123, 1360-1368. http://dx.doi.org/10.1542/peds.2008-2055

[4] Stoll, B.J., Hansen, N.I., Bell, E.F., Shankaran, S., Laptook, A.R., Walsh, M.C., et al. (2010) Neonatal Outcomes of Extremely Preterm Infants from the NICHD Neonatal Research Network. Pediatrics, 126, 443-456. http://dx.doi.org/10.1542/peds.2009-2959

[5] Clerihew, L., Lamagni, T.L., Brocklehurst, P. and McGuire, W. (2006) Invasive Fungal Infection in Very Low Birthweight Infants: National Prospective Surveillance Study. Archives of Disease in Childhood. Fetal and Neonatal Edition, 91, F188-F192. http://dx.doi.org/10.1136/adc.2005.082024

[6] Wang, M., Tran, Tu., Tran, V., Padilla, L. and Le Jennifer (2011) Case-Control Study of Risk Factors and Outcomes Associated with Neonatal Candidiasis. The Journal of Neonatal-Perinatal Medicine, 4, 39-43.

[7] Rodriguez, D., Almirante, B., Cuenca-Estrella, M., Rodriguez-Tudela, J.L., Mensa, J., Ayats, J., et al. (2010) Predictors of Candidaemia Caused by Candida Species Non-Albicans: Result of a Population-Based Surveillance in Barcelona, Spain. Clinical Microbiology and Infection, 16, 1666-1682. http://dx.doi.org/10.1111/j.1469-0691.2010.03208.x

[8] De Pauw, B., Walsh, T.J., Donnelly, J.P., Stevens, D.A., Edwards, J.E., Calandra, T., et al. (2008) Revised Definitions of Invasive Fungal Disease from European Organization for Research and Treatment of Cancer/Invasive Fungal Infections Cooperative Group and the National Institute of Allergy and Infectious Disease Mycoses Study Group (EORTC/ MSG) Consensus Group. Clinical Infectious Diseases, 46, 1813-1821. http://dx.doi.org/10.1086/588660

[9] Walsh, T.J., Groll, A., Hiemenz, J., Fleming, R., Roilidies, E. and Anaissie, E. (2004) Infections Due to Emerging and Uncommon Medically Important Fungal Pathogens. Clinical Microbiology and Infection, 10, 48-66. http://dx.doi.org/10.1111/j.1470-9465.2004.00839.x

[10] Roilides, E. and Walsh, T.J. (2010) Invasive Fungal Infection in Pediatric Patients. Clinical Microbiology and Infection, 16, 1319-1320. http://dx.doi.org/10.1111/j.1469-0691.2010.03337.x

[11] Neu, N., Malik, M., Lunding, A., Whittier, S., Alba, L., Kubin, C. and Saiman, L. (2009) Epidemiology of Candidemia at a Children's Hospital, 2002 to 2006. The Pediatric Infectious Disease Journal, 28, 806-809. http://dx.doi.org/10.1097/INF.0b013e3181a0d78d

[12] Figueiredo, G.C. and Tavares-Neto, J. (2001) Structuring a Database for Secondary Analysis of Information in Reports or Case Series. Revista Brasileira de Ortopedia, 36, 407-411.

[13] Hulley, S., Cummings, S.R., Browner, W.S., Grady, D.G. and Newman, T.B. (2008) Outlining Clinical Research, an Epidemiological Approach. 3th Edition, Guilford Press, New York.

[14] Amaral, P.M. and Tavares-Neto, J. (2010) Construction and Proposal of Evaluation Tools: A Biological Material for Occupational Health Personnel Exposure. Revista Brasileira de Saúde Ocupacional, 35, 131-147. http://dx.doi.org/10.1590/S0303-76572010000100015

[15] Amaral-Lopes, S. (2011) Fungemia in the Neonatal Period: A Secondary Data Analysis. Ph.D. Dissertation, Federal University of Bahia, Salvador.

[16] Figueiredo, G.C., Figueiredo, E.C.Q., Medeiros, A.K., Costa, F.A., Maia Junior, J.R. and Tavares-Neto, J. (2006) Fungal Osteomyelitis: A Secondary Analysis of Data. Revista Brasileira de Ortopedia, 41, 200-210.

[17] Figueiredo, G.C., Figueiredo, E.Q.C. and Tavares-Neto, J. (2001) Clinical and Therapeutic Aspects of Fungal Vertebral Osteomyelitis-Secondary Analysis of Data. Brazilian Journal of Rheumatology, 47, 34-44.

[18] Kirk, P.M. (2000) Species Fungorum (version 9.0, Sep 2010). In: Bisby, F.A., Roskov, Y.R., Orrell, T.M., Nicolson, D., Paglinawan, L.E., Bailly, N., Kirk, P.M., Bourgoin, T., Baillargeon, G. and Ouvrard, D., Eds., Species 2000 \& ITIS Catalogue of Life, 26th July 2011. http://www.catalogueoflife.org/col

[19] Gniadek, A., Macura, A.B., Twarużek, M. and Grajewski, J. (2010) Cytotoxicity of Aspergillus Strains Isolated from the Neonatal Intensive Care Unit Environment. Advances in Medical Sciences, 55, 242-249. 
http://dx.doi.org/10.2478/v10039-010-0039-y

[20] Sarvikivi, E., Lyytikäinen, O., Soll, D.R., Pujol, C., Pfaller, M.A., Richardson, M., Koukila-Kahkola, P., Luukkainen, P. and Saxén, H. (2005) Emergence of Fluconazole Resistance in a Candida Parapsilosis Strain That Caused Infections in the Neonatal Intensive Care Unit. Journal of Clinical Microbiology, 43, 2729-2735. http://dx.doi.org/10.1128/JCM.43.6.2729-2735.2005

[21] Manzoni, P., Jacqz-Aigrain, E., Rizzollo, S., Franco, C., Stronati, M., Mostert, M. and Farina, D. (2011) Antifungal Prophylaxis in Neonates. Early Human Development, 87, 59-60. http://dx.doi.org/10.1016/j.earlhumdev.2011.01.013

[22] Yildiran, A., Belet, N., Gunaydin, M. and Kuçuköduk, S. (2010) Comparison of Antifungal Susceptibilities in Candidemic Newborns According to Their Body Weights. Balkan Medical Journal, 27, 234-237.

[23] Pappas, P.G., Kauffman, C.A., Andes, D., Benjamin Jr., D.K., Calandra, T.F., Edwards Jr., J.E., et al. (2009) Clinical Practice Guidelines for the Management of Candidiasis: 2009 Update by the Infectious Diseases Society of America. Clinical Infectious Diseases, 48, 503-535. http://dx.doi.org/10.1086/596757

[24] Brecht, M. and Clerihew McGuire, L. (2009) Prevention and Treatment of Invasive Fungal Infection in Very Low Birthweight infants. Archives of Disease in Childhood. Fetal and Neonatal Edition, 94, 65-69. http://dx.doi.org/10.1136/adc.2007.133769

[25] Saiman, L., Ludington, E., Pfaller, M., Rangel-Frausto, S., Wiblin, R.T., Dawson, J., Blumberg, H.M., Patterson, J.E., Rinaldi, M., Edwards, J.E., Wenzel, R.P. and Jarvis, W. (2000) Risk Factors for Candidemia in Neonatal Intensive Care Unit Patients. The Pediatric Infectious Disease Journal, 19, 319-324. http://dx.doi.org/10.1097/00006454-200004000-00011

[26] Rodriguez, D., Almirante, B., Park, B.J., Cuenca-Estrella, M., Planes, A.M., Sanchez, F., Gene, A., Xercavins, M., Fontanals, D., Rodriguez-Tudela, J.L., Warnock, D.W. and Pahissa, A. (2006) Candidemia in Neonatal Intensive Care Units Barcelona, Spain. The Pediatric Infectious Disease Journal, 25, 224-229. http://dx.doi.org/10.1097/01.inf.0000202127.43695.06

[27] Moran, G.P., Coleman, D.C. and Sullivan, D.J. (2011) Comparative Genomics and the Evolution of Pathogenicity in Human Pathogenic Fungi. Eukaryotic Cell, 10, 34-42. http://dx.doi.org/10.1128/EC.00242-10

[28] Fernandez, J., Erstad, B.L., Petty, W. and Nix, D.E. (2009) Time to Positive Culture and Identification for Candida Bloodstream Infections. Diagnostic Microbiology and Infectious Disease, 64, 402-407. http://dx.doi.org/10.1016/j.diagmicrobio.2009.04.002

[29] Garey, K.W., Rege, M., Pai, M.P., Mingo, D.E., Suda, K.J., Turpin, R.S. and Bearden, D.T. (2006) Time to Initiation of Fluconazole Therapy Impacts Mortality in Patients with Candidemia: A multi-Institutional Study. Clinical Infectious Diseases, 43, 24-31. http://dx.doi.org/10.1086/504810

[30] Cho, J., Holditch-Davis, D. and Miles, M.S. (2010) Effects of Gender on the Health and Development of Medically At-Risk Infants. Journal of Obstetric, Gynecologic, \& Neonatal Nursing, 39, 536-549. http://dx.doi.org/10.1111/j.1552-6909.2010.01171.x 